\title{
Mapping the Chemistry Within, and the Strain Around, Al-alloy Precipitates at Atomic Resolution by Multi-frame Scanning Transmission Electron Microscopy
}

\author{
Lewys Jones ${ }^{1}$, Sigurd Wenner ${ }^{2}$, Magnus Nord ${ }^{2}$, Per Harald Ninive ${ }^{3}$, Ole Martin Løvvik ${ }^{4}$, \\ Calin D. Marioara ${ }^{4}$, Randi Holmestad ${ }^{2}$ and Peter D. Nellist ${ }^{1}$. \\ 1. Department of Materials, University of Oxford, Parks Road, OX1 3PH Oxford, UK \\ 2. Department of Physics, NTNU - Trondheim, NO-7491 Trondheim, Norway \\ 3. NTNU- Norwegian University of Science and Technology, Gjøvik, Norway, NO-2815 \\ 4. Materials and Chemistry, SINTEF, NO-7491 Trondheim, Norway
}

Al-Mg-Si(-Cu) alloys, used primarily in the transportation sector, are strengthened by nano-sized precipitate particles. There is a plethora of possible phases, depending on which elements are added to the alloy and how the material is heat treated. Here we present results from the $S, \beta$ ', $\beta$ ' $G e$ and Q' phases, which all form rod-shaped precipitate with typical dimensions 7x7x50 nm. These phases are meta-stable, which means that they cannot exist outside the Al matrix.

To fully understand the properties of these precipitates, both structural and chemical information is needed at the atomic scale. Fortunately both of these data are available using the scanning transmission electron microscope (STEM).

When viewed down a $\{100\}$ zone-axis, annular dark-field (ADF) images of rod-like precipitates can be recorded with sub-Angstrom resolution and the locations of atomic columns identified with picometre precision. However, obtaining high-quality atomic-resolution chemical maps is a compromise between collecting sufficient signal, while minimising sample damage from the electron dose. Classically the experimentalist has had only one parameter, beam current, to do this. Recently with the advent of fast and robust software tools for the non-rigid registration (scan-distortion correction) of multi-frame data [1], a new regime has opened up for the operator where both dose-per-frame and total-dose can be varied independently. Several studies now propose dose-rate as the critical parameter in minimising sample damage.

From high-speed multi-frame ADF image acquisition and processing we are able to map column positions with picometre scale precision (Figure 1). From such images lattice strains can be measured using either real- or Fourier-space methods (such as geometric phase analysis), which can be verified by density functional theory calculations (DFT). This method is now well established and can aid systematic studies across large numbers of precipitates with increased accuracy [2].

A similar approach was followed to obtain multi-scan EDX elemental maps at atomic resolution. The separate spectral volumes were aligned, corrected for scanning distortion and averaged. Figure 2, shows the experimental detectability of various elemental signals for the experimental conditions used and guides the experiment design. To increase the signal-to-noise ratio of weak elemental signals, the known symmetry of the precipitate phases was further exploited to yield 'symmetry averaged chemical maps'. These identify all the elements clearly for each type of precipitate without the need for noise filtering [3]. 
References: (Times New Roman, 12-pt, left justified)

[1] L. Jones et al. Adv. Struct. Chem. Imaging, 1:8, (2015) p. 1-16.

[2] S. Wenner and R. Holmestad, Scr. Mater., 118:7491, (2016) p. 5-8.

[3] The TEM work was carried out on the NORTEM (ARM200-CF), TEM Gemini Centre, NTNU,

Norway. SW and RH acknowledge the Norwegian Research Council, contract no 221714; LJ acknowledges the EU-FP7 Programme, Grant 312483: ESTEEM2.
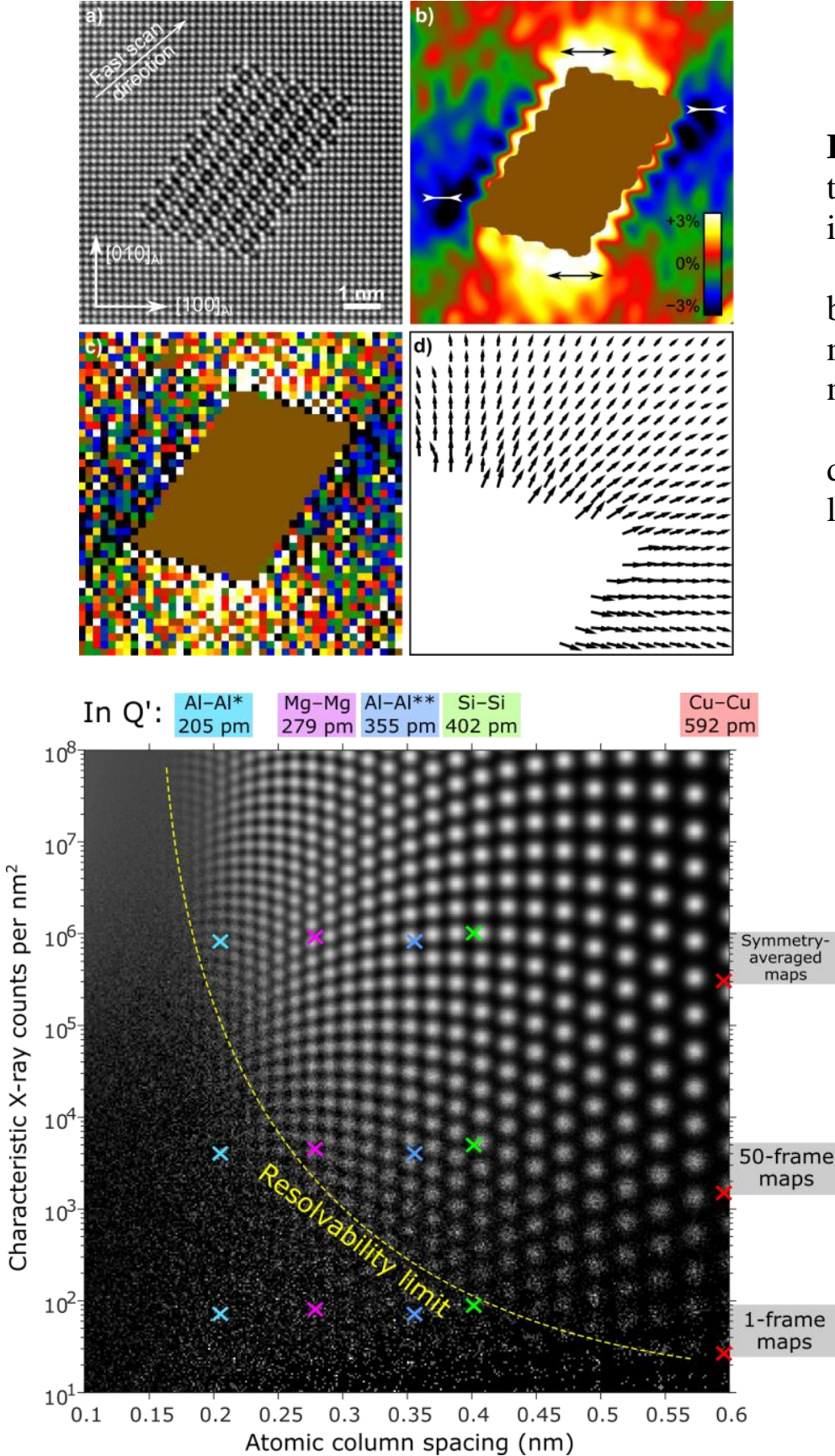

Figure 2. a) Average ADF image restored by the Smart Align algorithm. The field of view is about $1 / 8$ of the scan area.

b) $\varepsilon_{x x}$ plot measured using GPA with a $0.6 \mathrm{~nm}$ mask, and c) by real-space peak position measurement (same colour scale as b) ).

d) Atomic displacements from a perfect lattice in the upper right part of the image.

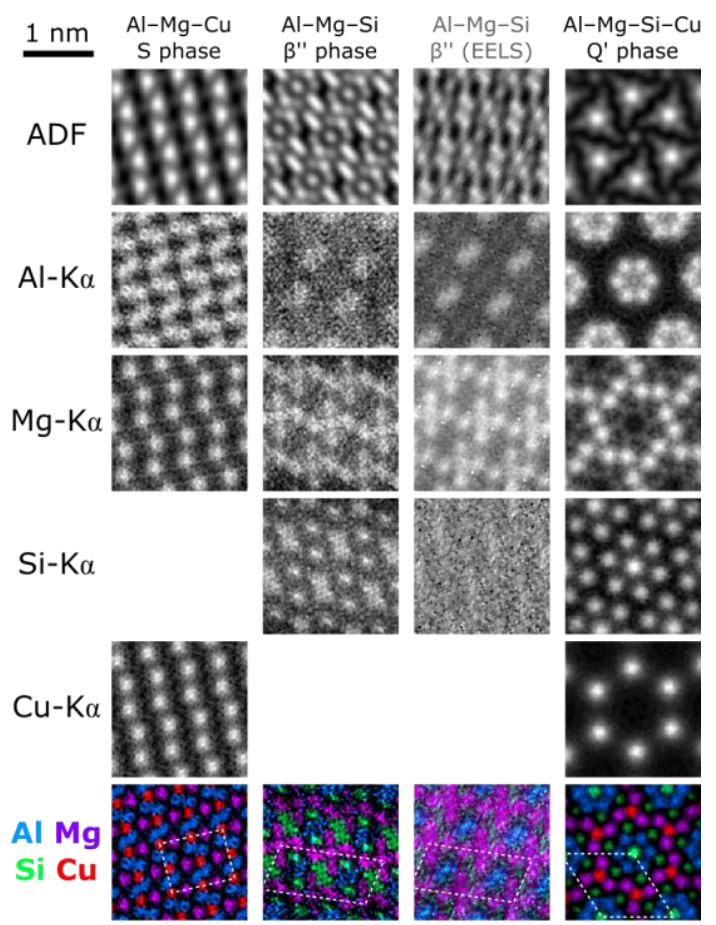

Figure 2. Left) experiment design planning for a JEOLARM200CF using a $155 \mathrm{pA}$ beam current, $500 \mu \mathrm{s}$ dwell-time and 0.98sr Centurio EDX detector. Right) experimental symmetry-averaged atomicresolution EDX maps from a range of $\mathrm{Al}-\mathrm{Mg}$ based rod-like precipitates. 